\title{
Use of Phonon Confinement Model in Simulation of Raman Spectra of Nanostructured Materials
}

\author{
M. Grujić-Brojčin*, M.J. Šćepanović, Z.D. Dohcević-Mitrović and Z.V. Popović \\ Center for Solid State Physics and New Materials, Institute of Physics \\ Pregrevica 118, Belgrade 11080, Serbia
}

\begin{abstract}
The simulation of the Raman spectra of nanostructured materials, where the effects of frequency shift and asymmetric broadening of the Raman modes play an important role, can be very useful in systematic characterization of these materials. Use of phonon confinement model for calculating Raman spectra of different nanomaterials is considered both from the viewpoint of different confinement function and the confinement strength, as well as the dimensionality of the confinement model. The phonon dispersion relations and the choice of their approximation are also studied. The influence of particle size distribution on the shape of the calculated spectra is discussed and contributions of Gaussian and asymmetric Gaussian distribution are compared. The effects of average and inhomogeneous strain on the behavior of simulated Raman spectra are also discussed. The results of the phonon confinement model are compared to the experimental spectra of $\mathrm{CeO}_{2}$ and anatase $\mathrm{TiO}_{2}$ nanopowders.
\end{abstract}

PACS numbers: 81.07.Wx, 78.30.-j, 63.22.-m, 07.05.Tp

\section{Introduction}

Phenomenological phonon confinement model (PCM) is widely used method in modeling Raman spectra of nanomaterials. For spherical particle of diameter $L$ and Gaussian confinement function, the resulting Raman intensity $I(\omega)$ can be presented as $[1,2]$ :

$$
I(\omega) \propto \sum_{i=1}^{m} \int_{0}^{\infty} \rho(L) \mathrm{d} L \int_{\mathrm{BZ}} \frac{\exp \left(\frac{-q^{2} L^{2}}{8 \beta}\right) \mathrm{d}^{3} q}{\left[\omega-\omega_{i}(q)\right]^{2}+(\Gamma / 2)^{2}},(1)
$$

where $\rho(L)$ is the particle size distribution (PSD), $q$ is wave vector in units of $\pi / a(a-$ unit cell parameter $)$, and $\Gamma$ is the intrinsic mode line width. The sum is carried over $m$ dispersion curves $\omega_{i}(q, T)$, depending on mode degeneration $m$.

\section{Results and discussion}

The confinement factor $\beta$ in literature often varies from $\beta=1$ in the Richter confinement model [1] to $\beta=2 \pi^{2}$ in the Campbell model [2], depending on confinement boundary conditions in different nanomaterials. Higher confinement factor leads to greater shift and broadening of the Raman spectrum, comparing to bulk (when $\beta \rightarrow 0)$.

The confinement dimension is also important factor in calculating Raman intensity, offering a lot of possibilities in modeling different types of nanomaterials [3].

\footnotetext{
* corresponding author; e-mail: myramyra@phy.bg.ac.yu
}

The three-dimensional (3D) PCM is appropriate for 0D objects such as powders, quantum dots, etc. where the infinitesimal volume element in Eq. (1) can be written as $\mathrm{d}^{3} q \propto q^{2} \mathrm{~d} q$. For $2 \mathrm{D}$ confinement (1D objects such as quantum wires, etc.) it is $\mathrm{d}^{3} q \propto q \mathrm{~d} q$ and for $1 \mathrm{D}$ confinement (2D objects - quantum wells, nanolayers etc.) $\mathrm{d}^{3} q \propto \mathrm{d} q$. With 3D confinement the broadening of Raman spectrum is the most pronounced [4].

To calculate the contributions of the optical phonons over a whole Brillouin zone (BZ) to the Raman spectra of nanoparticles, the phonon dispersion relations $\omega_{i}(q)$ are included in PCM. However, dispersion relations sometimes cannot be explicitly determined. In this way, the phonon dispersions for $E_{\mathrm{g}}$ mode of anatase $\mathrm{TiO}_{2}$ used by different authors [5-8] presented in Fig. 1a, show obvious discrepancy, especially away from center of BZ. Also, the choice of approximations for dispersion relations has great influence on the shape of the Raman spectrum. Two phonon dispersions of anatase $E_{\mathrm{g}}$ mode in $\Gamma-X$ direction, calculated by Mikami et al. [9], fitted by cosine, $\omega(q)=A+B(1-\cos q a)$, and polynomial, $\omega(q)=A+B_{1} q+B_{2} q^{2}+\ldots+B_{5} q^{5}$ functions, are shown in Fig. 1b. Corresponding Raman intensities are calculated along upper branch only (Fig. 1c) and both branches together (Fig. 1d). Although polynomial functions fit calculated phonon dispersions more precisely, the behaviour of both the linewidth and shape of corresponding Raman spectrum (Fig. 1(d)) suggests cosine dispersion as more proper for simulating Raman spectra of anatase $\mathrm{TiO}_{2}$ [8].

The Raman mode asymmetry is the result not only of phonon confinement in whole BZ, but also the PSD. In 


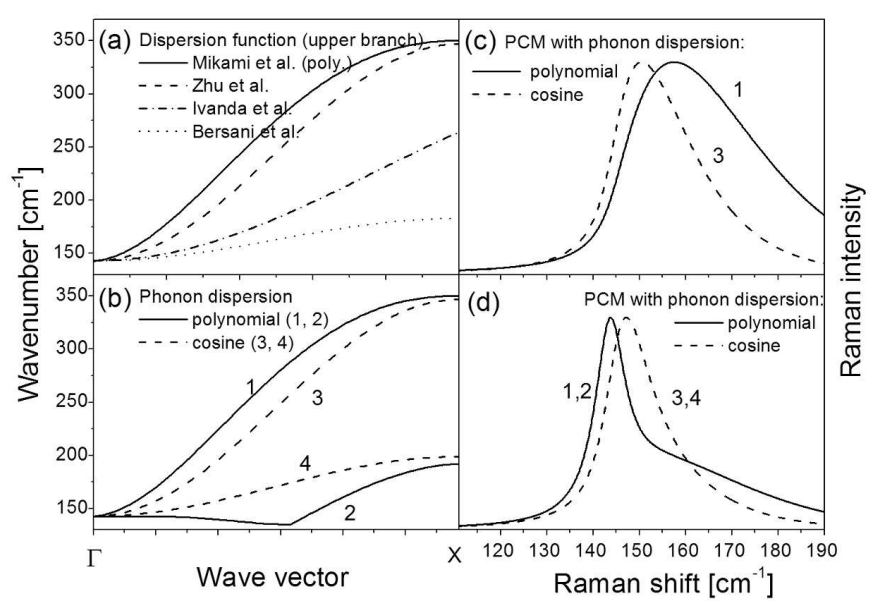

Fig. 1. The comparison of phonon dispersion functions for anatase $E_{\mathrm{g}}$ Raman mode in $\Gamma-X$ direction (a) published by different authors and (b) fitted by cosine (dashed line) and polynomial function (full line). $E_{\mathrm{g}}$ Raman mode intensity calculated by PCM using cosine (dashed line) and polynomial (full line) fit for dispersion functions: (c) upper dispersion branch only and (d) both branches in $\Gamma-X$ direction of BZ. Parameters of $\mathrm{TiO}_{2}: L_{0}=12 \mathrm{~nm}, \Gamma_{0}=8 \mathrm{~cm}^{-1}, \omega_{0}=144 \mathrm{~cm}^{-1}$, $\beta=2 \pi^{2}$.

Eq. (1) PSD is included by distribution function $\rho(L)$. Gaussian PSD $\rho(L)=(1 / \sigma \sqrt{2 \pi}) \exp \left(-\left(L-L_{0}\right)^{2} / 2 \sigma^{2}\right)$ is the most used distribution for nanoparticles [10], with $L_{0}$ as average particle size, and $\sigma$ as standard deviation (whereas FWHM is $w=2 \sigma \sqrt{\ln 4}$ ). In Fig. 2a the anatase $E_{\mathrm{g}}$ Raman mode calculated by PCM with symmetrical Gaussian PSD are shown.

If the results of some relevant measurement (scanning electron microscopy (SEM), transmission electron microscopy (TEM), atomic force micoscopy (AFM)) point out to asymmetric particle size distribution, then asymmetric Gaussian distribution can be used. It is given by

$$
\begin{aligned}
& \rho(L)=C / \sqrt{2 \pi \sigma} \cdot\left\{\left[1-h\left(L-L_{0}\right)\right]\right. \\
& \quad \times \exp \left(-\left(L-L_{0}\right)^{2} / 2 \sigma_{L}\right) \\
& \left.+h\left(L-L_{0}\right) \exp \left(-\left(L-L_{0}\right)^{2} / 2 \sigma_{D}\right)\right\},
\end{aligned}
$$

with $h\left(L-L_{0}\right)$ - step function, $L_{0}$ - particle size where asymmetric Gaussian has maximum, $\sigma_{L}$ and $\sigma_{D}$ - standard deviations corresponding to left and right side of the asymmetric curve, and $\sigma=\left(\sigma_{L}+\sigma_{D}\right) / 2$. In Fig. 2b anatase $E_{\mathrm{g}}$ Raman mode calculated by PCM with asymmetrical Gaussian PSD are shown. The distribution with $\sigma_{L}>\sigma_{D}$ increases mode asymmetry and shifts spectrum towards higher frequencies. Let us note that use of asymmetrical Gaussian PSD with proper choice of parameters gives similar results as using PCM with log-normal distribution [11].

The experimental Raman spectra of anatase $\mathrm{TiO}_{2}$

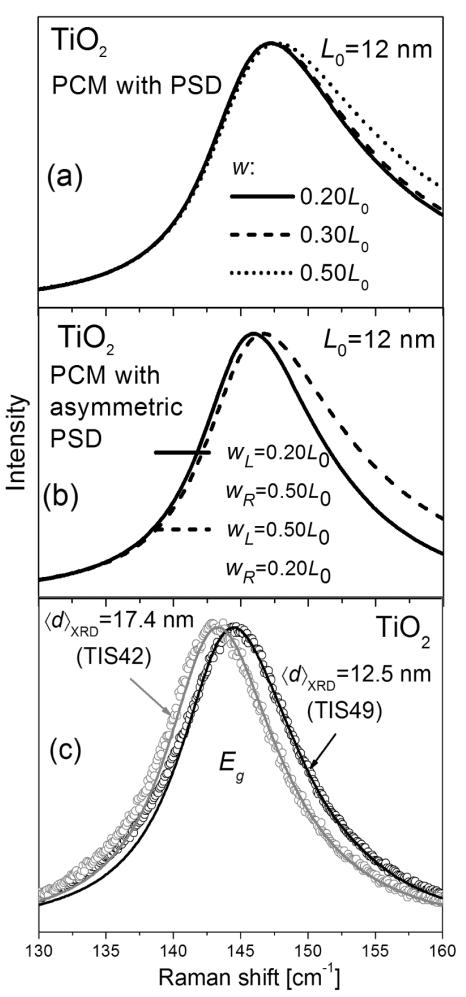

Fig. 2. $\quad E_{\mathrm{g}}$ Raman mode of anatase calculated by PCM with (a) symmetric and (b) asymmetric Gaussian PSD; (c) the experimental spectra of anatase nanopowders (circle) fitted by PCM (line) with asymmetric Gaussian PSD. Parameters: $\Gamma=8.5 \mathrm{~cm}^{-1}, \omega_{q=0}=142 \mathrm{~cm}^{-1}$, phonon dispersion calculated in cosine form, with the integration in all directions of $\mathrm{BZ}[8]$.

nanopowders presented by circles in Fig. 2c show the influence of particle size decreasing. It reflects through greater shift and broadening of Raman mode for sample with smaller nanoparticles, TIS49 (with mean particle size obtained from X-ray diffraction (XRD) data $\langle d\rangle_{\mathrm{XRD}}=12.5 \mathrm{~nm}$ ), in comparison to sample TIS42 (with $\left.\langle d\rangle_{\mathrm{XRD}}=17.4 \mathrm{~nm}\right)$. The results of PCM with asymmetric Gauss PSD with $w_{L} / w_{D}=0.25 / 0.60$ for TIS49, and $w_{L} / w_{D}=0.45 / 0.55$ for TIS42 (lines in Fig. 2c) show good agreement with experimental spectra.

The change of lattice parameters (lattice volume) with nanoparticle size $L$ decrease is registered in nanomaterials due to effect of microstrain $[12,13]$. To include the influence of strain on the Raman spectra, the dispersion function $\omega_{i}(q)$ in Eq. (1) must be replaced with $\omega_{i}(q)+\Delta \omega_{i}(q)$, where $\Delta \omega_{i}(q)$ is the term related to microstrain, given as $[10,14]$ :

$$
\Delta \omega_{i}(q)=-\gamma_{i} \omega_{i}(q) \Delta V / V_{0},
$$

where $\gamma$ is the Grüneisen parameter and $V$ volume of unit cell. In Fig. 3a the results of PCM with different kinds of strain for the Raman $F_{2 \mathrm{~g}}$ mode in $\mathrm{CeO}_{2}$ are shown. The dependence of lattice parameter $a$ in $\mathrm{CeO}_{2}$ cubic lattice is given by $a(L)=a_{0}+k / L^{2}$, where $a_{0}$ is 
bulk lattice parameter (Fig. 3a, inset), whereas $\gamma=1.23$ for $F_{2 \mathrm{~g}}$ mode in $\mathrm{CeO}_{2}$ [10]. When the PSD is uniform ( $L_{0}=$ const) PCM with so-called average strain included (dashed black line, Fig. 3a) gives mode with the same shape, but redshifted in comparison with mode obtained without strain (dashed gray line).

However, with nonuniform (Gaussian) PSD, the inhomogeneous strain is introduced in PCM, according to Eq. (3). It results both in frequency shift and pronounced asymmetric broadening of calculated Raman mode (black line). For cubic lattice, where $V=a^{3}$, Eq. (3) can be written as $\Delta \omega_{i}(q)=-3 \gamma_{i} \omega_{i}(q) \Delta a / a_{0}$. In Fig. 3b the results of PCM are compared with the experimental Raman spectrum of $\mathrm{CeO}_{2-\delta}$ nanopowder. The Raman $F_{2 \mathrm{~g}}$ mode calculated by PCM with inhomogeneous strain, symmetric Gaussian PSD $\left(L_{0}=7 \mathrm{~nm}, w=0.52 L_{0}\right)$ and phonon confinement $\beta=4 \pi^{2}$ [14] (full line), fits the broadening of experimental spectrum better than PCM with average (dots), or inhomogeneous (dashed line) strain with $\beta=2 \pi^{2}[2,10]$.
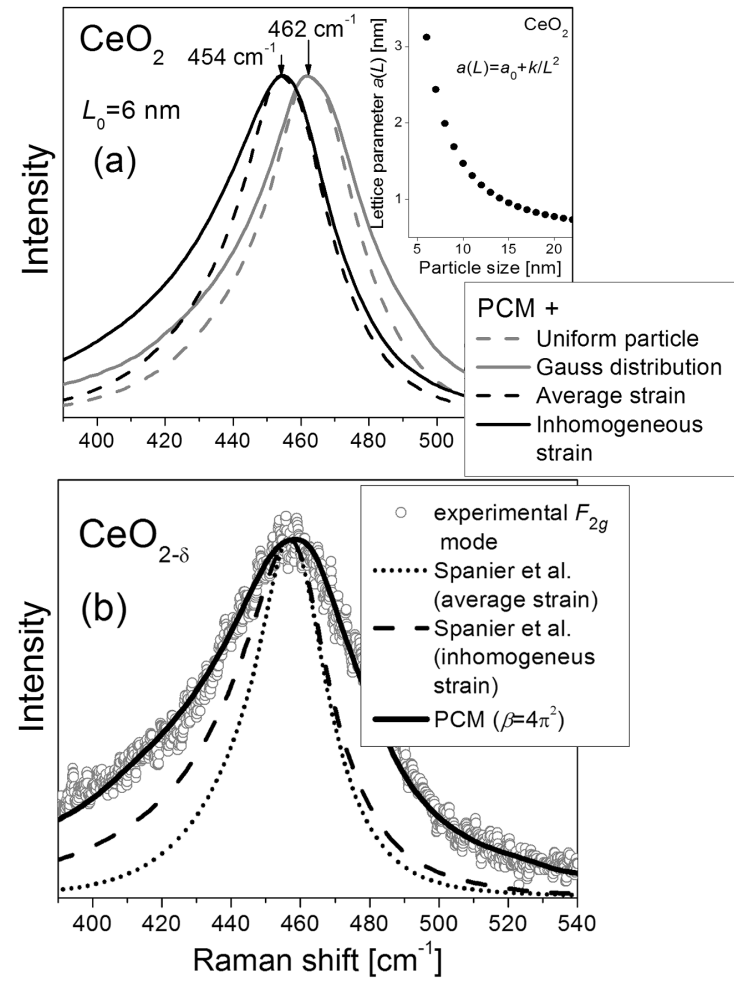

Fig. 3. (a) The comparison of PCM results for Raman $F_{2 \mathrm{~g}}$ mode in $\mathrm{CeO}_{2-\delta}$ nanopowder with $L_{0}=6 \mathrm{~nm}$ (inset: the lattice parameter vs. particle size [10, 14]); (b) experimental and PCM results for $F_{2 \mathrm{~g}}$ mode in $\mathrm{CeO}_{2-\delta}$.

Opposite to $\mathrm{CeO}_{2}$, the lattice parameters of tetragonal anatase $\mathrm{TiO}_{2}$ decrease with nanopartical size decreasing (Fig. 4b) due to compressive strain [12, 15]. Therefore, the unit cell volume can be approximated by: $V(L)=V_{0}-k_{1} / L^{k_{2}}$, with bulk unit cell volume $V_{0}=a_{0}^{2} c_{0}$, and adjustable parameters $k_{1}$ and $k_{2}$ [15].
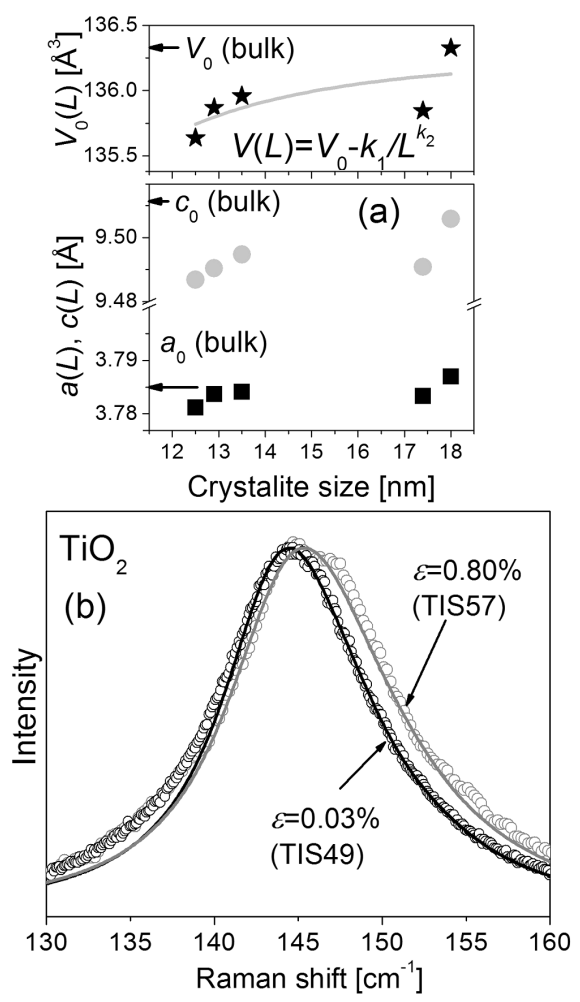

Fig. 4. (a) Lattice parameters $(a, c)$ and unit cell volume $(V)$ vs. particle size from XRD measurements [15]. (b) Experimental spectra of $E_{\mathrm{g}}$ Raman mode (circles) with PCM results (lines) for anatase $\mathrm{TiO}_{2}$ nanopowders.

The experimental spectra of $\mathrm{TiO}_{2}$ nanopowders (TIS49 and TIS57) with different strain values $\varepsilon_{X R D}$, obtained from XRD analyses, together with the results of PCM are shown in Fig. 4. Fitted spectrum for TIS49 with small strain value $\left(\varepsilon_{\mathrm{XRD}}=0.03 \%\right)$ is obtained by PCM with no strain included (as in Fig. 2), whereas appropriate blueshift of $E_{\mathrm{g}}$ mode caused by relatively great strain $\left(\varepsilon_{\mathrm{XRD}}=0.80 \%\right)$ in TIS57 sample is simulated by PCM with inhomogeneous strain, calculated by Eq. (3) with Grüneisen parameter for anatase $E_{\mathrm{g}}$ mode $\gamma_{i}=4.23$ [15].

\section{Conclusion}

The results of phonon confinement model are compared to the experimental spectra of $\mathrm{CeO}_{2}$ and anatase $\mathrm{TiO}_{2}$ nanopowders. It is shown that proper choice of confinement parameters, phonon dispersion relations, particle size distribution and the strain type (tensile or compressive), in accordance with physical properties of the nanomaterials allows precise modeling of their Raman spectra. This confirms use of the phonon confinement model in simulation of the Raman spectra of nanostructured materials as favorable method in estimation of their nanostructural characteristics. 


\section{Acknowledgments}

This work is supported by the Serbian Ministry of Science and Technological Development under project No. 141047 and the OPSA-026283 project within the EC FP6 program.

\section{References}

[1] H. Richter, Z.P. Wang, L. Ley, Solid State Commun. 39, 625 (1981).

[2] I.I. Campbell, P.M. Fauchet, Solid State Commun. 58, 739 (1986).

[3] A.K. Arora, M. Rajalakshmi, T. R. Ravindran, V. Sivasubramanian, J. Raman Spectrosc. 38, 604 (2007).

[4] M.J. Šćepanović, M. Grujić-Brojčin, Z. DohčevićMitrović, Z.V. Popović, J. Optoel. Adv. Mater. 9, 178 (2007).

[5] D. Bersani, P.P. Lottici, X.Z. Ding, Appl. Phys. Lett. 72, 73 (1998).

[6] M. Ivanda, S. Musić , M. Gotić , A. Turković , A.M. Tonejc, O. Gamulin, J. Mol. Struct. 480-481, 641 (1999).
[7] K.R. Zhu, M.S. Zhang, Q. Chen, Z. Yin, Phys. Lett. A 340, 220 (2005).

[8] M.J. Šćepanović, M. Grujić-Brojčin, Z. DohčevićMitrović, Z.V. Popović, Appl. Phys. A 86, 365 (2007).

[9] M. Mikami, S. Nakamura, O. Kitao, H. Arakawa, Phys. Rev. B 66, 1552131 (2002).

[10] J.E. Spaneir, R.D. Robinson, F. Zhang, S.-W. Chan, I.P. Herman, Phys. Rev. B 64, 2454071 (2001).

[11] M. J. Šćepanović, M. Grujić-Brojčin, Z. DohčevićMitrović, Z.V. Popović, Sci. Sintering 41, 67 (2009).

[12] V. Swamy, D. Menzies, B.C. Muddle, A. Kuznetsov, L.S. Dubrovinsky, Appl. Phys. Lett. 88, 243103 (2006).

[13] F. Zhang, S.-W. Chan, J.E. Spanier, E. Apak, Q. Jin, R.D. Robinson, I.P. Herman, Appl. Phys. Lett. 80, 127 (2002).

[14] Z.D. Dohčević-Mitrović, M.J. ćepanović, M.U. GrujićBrojčin, Z.V. Popović, S.B. Boković, B.M. Matović, M.V. Zinkevich, F. Aldinger, Solid State Commun. 137, 387 (2006).

[15] M.J. Šćepanović, M.U. Grujić-Brojčin, Z.D. Dohčević-Mitrović, Z.V. Popović, Mater. Sci. Forum 518, 101 (2006). 